\title{
Beating pulmonary vein sign during catheter ablation
}

\author{
Shinichi Ishida ${ }^{1}$, Yoshio Takemoto ${ }^{1}$, Yuichiro Yamase $^{1}$, and Kei Yagami ${ }^{1}$ \\ ${ }^{1}$ Gifu Prefectural Tajimi Hospital
}

September 25, 2021

\begin{abstract}
The beating of a pulmonary vein during cardiac catheterization is a rare phenomenon caused by the heart beating through the pericardial effusion when a cardiac tamponade occurs. This "beating pulmonary vein" sign is useful for early detection of a tamponade before circulatory collapse occurs.
\end{abstract}

Title: Beating pulmonary vein sign during catheter ablation

Authors:

Shinichi Ishida $\mathrm{MD}^{1}$, Yoshio Takemoto MD-PhD ${ }^{2}$, Yuichiro Yamase $\mathrm{MD}^{2}$, Kei Yagami MD-PhD ${ }^{1}$

${ }^{1}$ Department of Cardiac Surgery, Gifu Prefectural Tajimi Hospital, Tajimi, Gifu, Japan

${ }^{2}$ Department of Cardiovascular Medicine, Gifu Prefectural Tajimi Hospital, Tajimi, Gifu, Japan

Conflicts of interest: The authors declare that there are no conflicts of interests.

Financial disclosures: The authors received no financial support for the preparation of this article.

Acknowledgment: No relevant acknowledgments.

Corresponding Author: Shinichi Ishida, 5-161 Maebata-cho, Tajimi-city, Gifu 507-8522, Japan, +81-572-22-

5311, e-mail:shin1dinho@yahoo.co.jp

Key clinical message:

The "beating pulmonary vein" sign during cardiac catheterization is useful for early detection of a tamponade before circulatory collapse occurs. Moreover, we need to be careful when finding the beating of venous system that does not usually beat.

Abstract:

The beating of a pulmonary vein during cardiac catheterization is a rare phenomenon caused by the heart beating through the pericardial effusion when a cardiac tamponade occurs. This "beating pulmonary vein" sign is useful for early detection of a tamponade before circulatory collapse occurs.

Key words: Cardiac tamponade, Cardiac catheterization, Beating pulmonary vein

Clinical presentation:

A 71-year-old man with a history of paroxysmal atrial fibrillation underwent cryoballoon catheter ablation. Immediately after undergoing left superior pulmonary vein (LSPV) ablation, his blood pressure decreased to $45 / 35 \mathrm{mmHg}$. Thoracic echocardiography revealed a tamponade, which was immediately treated with drainage by needle paracentesis. The patient's hemodynamic state stabilized after pericardial blood drainage 
with inotropic drug support and blood transfusion. The laceration site was assumed to be at the left atrium roof close to the LSPV during transatrial puncture with the cryoballoon sheath.

The angiography recorded while the LSPV was occluded using a cryoballoon showed the LSPV to be already beating before a decline in blood pressure (Figure 1, Video 1), which was identified retrospectively. This phenomenon was presumably caused by the heart beating through the pericardial effusion. This "beating PV" sign is useful for early detection of a tamponade before circulatory collapse occurs.

Figure caption:

Figure 1. Angiogram showing synchronized beating of the left superior pulmonary vein (arrow) and heart. A, Systole. B, Diastole.

Video 1. Angiography performed during ablation of the left superior pulmonary vein. The beating of the left superior pulmonary vein is synchronized with the heartbeat.

Ethical statement:

Institutional review board approval was exempted at our institution for this retrospectively designed report and no informed consent is applicable given that no identifying information was used.

Author contributions:

SI: wrote the draft of the manuscript and prepared the figures. SI, HT and YY: involved in writing. SI, HT and KY: revised and approved the final manuscript.
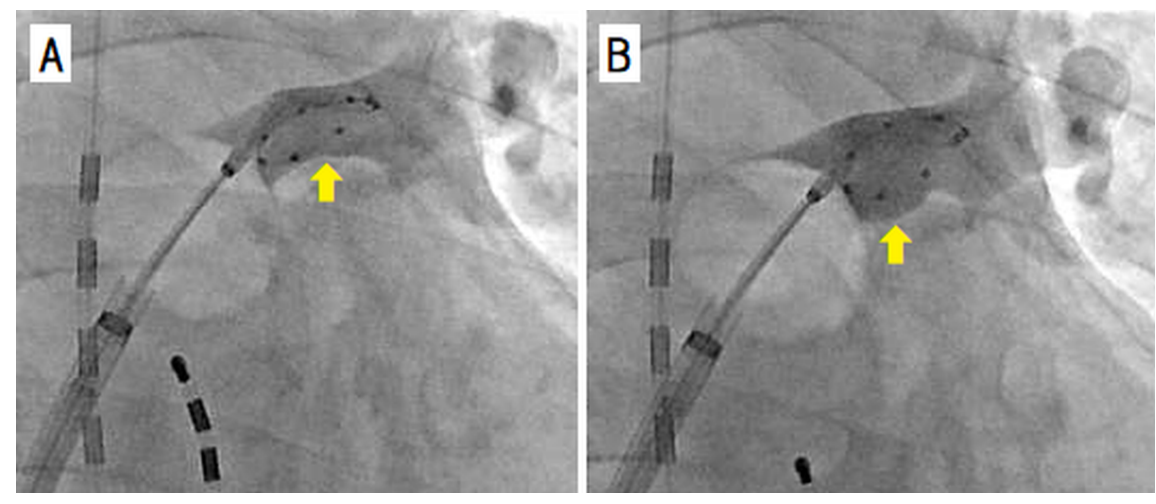